Discussion Paper No. 961

\title{
STRATEGY-PROOFNESS \\ ON BANKRUPTCY PROBLEMS WITH AN INDIVISIBLE OBJECT
}

\author{
Kazuhiko Hashimoto \\ Yu Nakayama
}

February 2016

The Institute of Social and Economic Research Osaka University

6-1 Mihogaoka, Ibaraki, Osaka 567-0047, Japan 


\title{
Strategy-Proofness on Bankruptcy Problems with an Indivisible Object
}

\author{
Kazuhiko Hashimoto* \\ Institute of Social and Economic Research, Osaka University
}

and

Yu Nakayama ${ }^{\dagger}$

Graduate School of Economics, Osaka University

February 27, 2016

\begin{abstract}
We analyze bankruptcy problems with an indivisible object, where real owners and outside traders want to allocate an indivisible object among them with monetary compensation. The object might be a company that has gone bankrupt or a house left by a parent who has died, and so on. We show that there exists no rule satisfying strategyproofness and the ownership lower bound on any domains that include at least three common preferences.
\end{abstract}

Keywords: Strategy-proofness; Ownership lower bound; Equal right lower bound; Impossibility result; Finitely restricted preference domains.

JEL codes: D47; D71.

*Corresponding address: Institute of Social and Economic Research, Osaka University, 6-1 Mihogaoka, Ibaraki, Osaka, 567-0047, JAPAN. E-mail: k-hashimoto@iser.osaka-u.ac.jp

${ }^{\dagger}$ Corresponding address: Graduate School of Economics, Osaka University, 1-7, Machikaneyama, Toyonaka, Osaka, 560-0043, JAPAN. E-mail: sge015ny@student.econ.osaka-u.ac.jp 


\section{Introduction}

We analyze bankruptcy problems "with an indivisible object." "Normal" bankruptcy problems consider how to allocate an amount of remaining money among owners of a company that has gone bankrupt according to their claims, such as portions of ownership. A rule is a function that associates monetary allocation with claims. The claims are private information and owners might try to manipulate a rule by reallocating their claims within a group in advance. Hence, one of the main purposes of analyses is to design a rule that is immune to such manipulation. This non-manipulability condition is called reallocation-proofness [Moulin (1985)]. ${ }^{1}$

On the other hand, bankruptcy problems with an indivisible object consider how to allocate an indivisible object ${ }^{2}$ such as a factory or a company among owners ${ }^{3}$ with monetary compensation according to their preferences. Their portions of ownership are public information. A rule is a function that associates object assignment and monetary compensation with preferences. Their preferences are private information and owners might try to manipulate a rule by misrepresenting their preferences. Hence, a primary purpose of analysis is to design a rule that is immune to such manipulation. This non-manipulability condition is called strategy-proofness [Gibbard (1973) and Satterthwaite (1975)]. ${ }^{4}$

In addition to strategy-proofness, we study rules satisfying a voluntary participation condition called the ownership lower bound [Dubins and Spanier (1961) and Cramton et al. (1987)]. Imagine a situation where an owner whose portion of ownership is $30 \%$ evaluates the object to be worth $\$ 1,000$. When the owner receives compensation less than $\$ 300$ in spite of giving up his ownership of the object, he does not admit this decision. Similarly, when he receives the object but must pay more than $\$ 700$, he does not admit this decision. To make him admit the decision of the rule, the rule must make his utility level at least $\$ 300$. Formally, the ownership lower bound requires that each owner's utility under the rule be at least as much as his value for the object multiplied by his portion of ownership. When portions of ownership are equal among owners, this condition is equivalent to the equal right lower bound [Steinhaus (1948) and Moulin (1992)].

\footnotetext{
${ }^{1}$ See Moulin (2002) and Thomson (2003, 2014) for surveys.

${ }^{2}$ We allow the object to be bad. Then, the problem is referred to as a "NIMBY problem." See, for example, Sakai (2012) and Fujinaka (2008).

${ }^{3}$ We admit that there are not only owners whose portions of ownership are positive, but also owners whose portions of ownership are zero. Strictly speaking, the former are real owners, and the latter are outside traders.

${ }^{4}$ See Sprumont (1995) and Barberà (2001, 2012) for surveys.
} 
Bankruptcy problems with an indivisible object have other important applications. For example, consider an "inheritance problem," as follows. An indivisible object is a house left by a parent who has died. Owners might be his wife and children. ${ }^{5}$ Their portions of ownership are determined by law, in advance. They must decide who inherits the house with monetary compensation. How should they do so? This is an issue that has caused problems in family estates all over the world.

We show that there exists no rule satisfying strategy-proofness and the ownership lower bound. ${ }^{6}$ Furthermore, this impossibility result is valid on any domains that include at least three common preferences. ${ }^{7}$ Thus, it is important to consider other lower bounds or to expand the research scope from deterministic rules to probabilistic rules. ${ }^{8}$

The rest of this paper is organized as follows. Section 2 sets up the model. Section 3 introduces the axioms. Section 4 states the results. Lastly, Section 5 provides proof.

\section{Model}

We consider an environment with a single indivisible object, hereafter called object, and one divisible good called money. The object might be a company that has gone bankrupt or a house left by a parent who has died, and so on. Let $N=\{1,2, \ldots, n\}$ be the set of agents, where we assume $n \geq 2$. We denote agent $i$ 's portion of ownership for the object by $\alpha_{i} \geq 0$, which is public information. Notice that we allow $\alpha_{i}=0$ for some $i \in N .^{9}$ We assume that for at least two agents, their portions of ownership are positive. We call $\left(\alpha_{i}\right)_{i \in N}$ such that $\sum_{i \in N} \alpha_{i}=1$, an ownership profile.

Each agent $i \in N$ has a preference over bundles consisting of object assignment $x_{i} \in\{0,1\}$ and monetary assignment $m_{i} \in \mathbb{R}$. We assume that this preference is represented by a utility function $u_{i}\left(x_{i}, m_{i}\right)=v_{i} x_{i}+m_{i}$ for

\footnotetext{
${ }^{5}$ Of course, outside traders can also be participants.

${ }^{6}$ Moulin (2010) has shown that there exists no rule satisfying strategy-proofness and the equal right lower bound on a continuous domain.

${ }^{7}$ Almost all studies on mechanism design assume preference domains that have cardinality of the continuum. For example, see Holmström (1979), Zhou (1990), and Serizawa (2002). However, a real economy may be finite. Hence, it is important to analyze problems on finitely restricted preference domains. See also Ohseto (2000), Ando et al. (2008), and Kato et al. (2015).

${ }^{8}$ See, for example, Porter et al. (2004) for a study on other lower bounds and Hashimoto (2015) for a possibility result when we allow probabilistic rules.

${ }^{9}$ In other words, $N$ can include not only real owners but also outside traders.
} 
some $v_{i} \in V_{i} \subset \mathbb{R}$. Since a preference ${ }^{10}$ is identified by $v_{i}$, we regard $v_{i}$ and $V_{i}$ as the preference and the set of preferences, respectively. We assume that $v_{i}$ is private information of agent $i \in N$. We call a list $v \equiv\left(v_{i}\right)_{i \in N} \in \prod_{i \in N} V_{i}$ a preference profile.

The set of feasible allocations is

$$
Z=\left\{\left(x_{i}, m_{i}\right)_{i \in N} \in(\{0,1\} \times \mathbb{R})^{n}: \sum_{i \in N} x_{i}=1 \text { and } \sum_{i \in N} m_{i} \leq 0\right\}
$$

A rule is a function $f: \Pi_{i \in N} V_{i} \rightarrow Z$. Given a rule $f$ and a preference profile $v \in \Pi_{i \in N} V_{i}$, we denote by $f_{i}(v) \equiv\left(x_{i}(v), m_{i}(v)\right) \in\{0,1\} \times \mathbb{R}$, agent $i$ 's assignment under $f(v)$. For any $v \in \prod_{i \in N} V_{i}$ and $N^{\prime} \subseteq N$, let $v_{N^{\prime}} \in \Pi_{i \in N^{\prime}} V_{i}$ and $v_{-N^{\prime}} \in \prod_{i \in N \backslash N^{\prime}} V_{i}$ denote $\left(v_{j}\right)_{j \in N^{\prime}}$ and $\left(v_{j}\right)_{j \notin N^{\prime}}$, respectively.

\section{Axioms}

We introduce the desirable properties. First, strategy-proofness states that it is a dominant strategy for any agent to report his true preference.

Definition 1. A rule $f$ satisfies strategy-proofness if for any $v \in \prod_{i \in N} V_{i}$, any $i \in N$, and any $v_{i}^{\prime} \in V_{i}$, it holds that

$$
v_{i} x_{i}(v)+m_{i}(v) \geq v_{i} x_{i}\left(v_{i}^{\prime}, v_{-i}\right)+m_{i}\left(v_{i}^{\prime}, v_{-i}\right) .
$$

Next, the ownership lower bound states that each owner's utility under the rule is at least as much as his value for the object multiplied by his portion of ownership.

Definition 2. A rule $f$ satisfies the ownership lower bound if for any $v \in \Pi_{i \in N} V_{i}$ and any $i \in N$, it holds that

$$
v_{i} x_{i}(v)+m_{i}(v) \geq \alpha_{i} v_{i}
$$

When for any $i \in N, \alpha_{i}=\frac{1}{n}$, the ownership lower bound is equivalent to the equal right lower bound, defined below.

Definition 3. A rule $f$ satisfies the equal right lower bound if for any $v \in \Pi_{i \in N} V_{i}$ and any $i \in N$, it holds that

$$
v_{i} x_{i}(v)+m_{i}(v) \geq \frac{1}{n} v_{i}
$$

\footnotetext{
${ }^{10}$ Notice that we also allow negative valuations.
} 


\section{Results}

We state the results. The first result states that no rule satisfies the axioms on domains where agents have three common preferences. The proof is provided in next section.

Theorem 1. Given any ownership profile $\left(\alpha_{i}\right)_{i \in N}$. Let $V_{1}, V_{2}, \ldots, V_{n}$ be such that $V_{1}=V_{2}=\cdots=V_{n}$ and $\# V_{i}=3$. Then, there exists no rule satisfying strategy-proofness and the ownership lower bound.

Since an impossibility result is also valid on larger domains, from this theorem, we immediately have the next result, which states that no rule satisfies the axioms on domains that include at least three common preferences.

Corollary 1. Given any ownership profile $\left(\alpha_{i}\right)_{i \in N}$. Let $V_{1}, V_{2}, \ldots, V_{n}$ be such that $\#\left(\cap_{i \in N} V_{i}\right) \geq 3$. Then, there exists no rule satisfying strategy-proofness and the ownership lower bound.

The following corollary is a generalization of Moulin (2010), who has shown the impossibility on a continuous domain.

Corollary 2. Let $V_{1}, V_{2}, \ldots, V_{n}$ be such that $\#\left(\cap_{i \in N} V_{i}\right) \geq 3$. Then, there exists no rule satisfying strategy-proofness and the equal right lower bound.

Since the independence of the axioms in Theorem 1 is trivial, we omit the details here.

\section{Proof of Theorem 1}

Lemma 1. Let $f$ be a rule satisfying strategy-proofness. For any $v \in \Pi_{i \in N} V_{i}$, any $i \in N$, and any $v_{i}^{\prime} \in V_{i}$, it holds that

1. if $x_{i}(v)=1$ and $v_{i}<v_{i}^{\prime}$, then $f_{i}(v)=f_{i}\left(v_{i}^{\prime}, v_{-i}\right)$,

2. if $x_{i}(v)=0$ and $v_{i}>v_{i}^{\prime}$, then $f_{i}(v)=f_{i}\left(v_{i}^{\prime}, v_{-i}\right)$.

Proof of Lemma 1. We show the first part. The second part is similar, so we omit it here. Let $v \in \Pi_{i \in N} V_{i}$. Let $i \in N$ be such that $x_{i}(v)=1$. Let $v_{i}^{\prime} \in V_{i}$ be such that $v_{i}<v_{i}^{\prime}$. By strategy-proofness, it holds that

$$
\left\{\begin{array}{l}
v_{i} x_{i}(v)+m_{i}(v) \geq v_{i} x_{i}\left(v_{i}^{\prime}, v_{-i}\right)+m_{i}\left(v_{i}^{\prime}, v_{-i}\right) \\
v_{i}^{\prime} x_{i}\left(v_{i}^{\prime}, v_{-i}\right)+m_{i}\left(v_{i}^{\prime}, v_{-i}\right) \geq v_{i}^{\prime} x_{i}(v)+m_{i}(v),
\end{array}\right.
$$


that is,

$$
\left\{\begin{array}{l}
v_{i}\left(1-x_{i}\left(v_{i}^{\prime}, v_{-i}\right)\right) \geq m_{i}\left(v_{i}^{\prime}, v_{-i}\right)-m_{i}(v) \\
m_{i}\left(v_{i}^{\prime}, v_{-i}\right)-m_{i}(v) \geq v_{i}^{\prime}\left(1-x_{i}\left(v_{i}^{\prime}, v_{-i}\right)\right),
\end{array}\right.
$$

which implies that

$$
v_{i}\left(1-x_{i}\left(v_{i}^{\prime}, v_{-i}\right)\right) \geq v_{i}^{\prime}\left(1-x_{i}\left(v_{i}^{\prime}, v_{-i}\right)\right),
$$

that is,

$$
\left(v_{i}-v_{i}^{\prime}\right)\left(1-x_{i}\left(v_{i}^{\prime}, v_{-i}\right)\right) \geq 0 .
$$

Since $v_{i}<v_{i}^{\prime}$, it must be that

$$
x_{i}\left(v_{i}^{\prime}, v_{-i}\right)=1 .
$$

Hence, we have $x_{i}(v)=x_{i}\left(v_{i}^{\prime}, v_{-i}\right)$. Then, by strategy-proofness, we also obtain that $m_{i}(v)=m_{i}\left(v_{i}^{\prime}, v_{-i}\right)$. Therefore, we have $f_{i}(v)=f_{i}\left(v_{i}^{\prime}, v_{-i}\right)$.

Lemma 2. Let $f$ be a rule satisfying the ownership lower bound. Let $v \in$ $\Pi_{i \in N} V_{i}$. Let $i \in N$ be such that $v_{i}=\min \left\{v_{1}, v_{2}, \ldots, v_{n}\right\}$. If there exists $j \in N$ such that $\alpha_{j}>0$ and $v_{j}>v_{i}$, then we have $x_{i}(v)=0$.

Proof of Lemma 2. Let $j \in N$ be such that $\alpha_{j}>0$ and $v_{j}>v_{i}$. Suppose, to the contrary, that $x_{i}(v)=1$. By the ownership lower bound, we obtain that

$$
m_{i}(v) \geq \alpha_{i} v_{i}-v_{i}=-\left(1-\alpha_{i}\right) v_{i}
$$

and

$$
m_{j}(v) \geq \alpha_{j} v_{j}>\alpha_{j} v_{i},
$$

and also that, for any $k \neq i, j$,

$$
m_{k}(v) \geq \alpha_{k} v_{k} \geq \alpha_{k} v_{i}
$$

By summing these inequalities, we have

$$
\sum_{h \in N} m_{h}(v)>-\left(1-\alpha_{i}\right) v_{i}+\sum_{h \neq i} \alpha_{h} v_{i}=0 .
$$

This contradicts the feasibility. Therefore, we have $x_{i}(v)=0$.

Proof of Theorem 1. Suppose, to the contrary, that there exists a rule $f$ on $\prod_{i \in N} V_{i}$ satisfying strategy-proofness and the ownership lower bound. Denote $V_{i}=\{a, b, c\}$, where $a<b<c$. Without loss of generality, we may assume that $x_{1}(a, a, \ldots, a)=1$. 
Claim 1. $f_{1}(a, a, \ldots, a)=\left(1,-\left(1-\alpha_{1}\right) a\right)$, and for any $i \neq 1, f_{i}(a, a, \ldots, a)=$ $\left(0, \alpha_{i} a\right)$.

Proof of Claim 1. By the ownership lower bound, we obtain that

$$
m_{1}(a, a, \ldots, a) \geq-\left(1-\alpha_{1}\right) a
$$

and that, for any $i \neq 1$,

$$
m_{i}(a, a, \ldots, a) \geq \alpha_{i} a
$$

Then, by the feasibility, these imply that

$$
m_{1}(a, a, \ldots, a)=-\left(1-\alpha_{1}\right) a
$$

and that, for any $i \neq 1$,

$$
m_{i}(a, a, \ldots, a)=\alpha_{i} a
$$

which are the desired results.

Claim 2. $f_{1}(b, a, \ldots, a)=\left(1,-\left(1-\alpha_{1}\right) a\right)$, and for any $i \neq 1, f_{i}(b, a, \ldots, a)=$ $\left(0, \alpha_{i} a\right)$.

Proof of Claim 2. Since $b>a$, by Claim 1 and Lemma 1, we have

$$
f_{1}(b, a, \ldots, a)=f_{1}(a, a, \ldots, a)=\left(1,-\left(1-\alpha_{1}\right) a\right) .
$$

Then, by the ownership lower bound, for any $i \neq 1$, it follows that

$$
m_{i}(b, a, \ldots, a) \geq \alpha_{i} a,
$$

which means, by the feasibility, that

$$
m_{i}(b, a, \ldots, a)=\alpha_{i} a .
$$

These are the desired results.

In the following, without loss of generality, we assume that $\alpha_{2}>0$.

Claim 3. $x_{2}(b, b, a, \ldots, a)=1$ and $m_{2}(b, b, a, \ldots, a) \geq-\left(1-\alpha_{2}\right) b$. 
Proof of Claim 3. Suppose, to the contrary, that $x_{2}(b, b, a, \ldots, a)=0$. Since, by Claim $2, f_{2}(b, a, a, \ldots, a)=\left(0, \alpha_{2} a\right)$, it holds, by strategy-proofness, that

$$
m_{2}(b, b, a, \ldots, a)=m_{2}(b, a, a, \ldots, a)=\alpha_{2} a .
$$

However, the ownership lower bound requires that

$$
m_{2}(b, b, a, \ldots, a) \geq \alpha_{2} b>\alpha_{2} a,
$$

which is a contradiction. Thus, we obtain that

$$
x_{2}(b, b, a, \ldots, a)=1 .
$$

Then, by the ownership lower bound, we have

$$
m_{2}(b, b, a, \ldots, a) \geq-\left(1-\alpha_{2}\right) b .
$$

Claim 4. $x_{2}(b, c, a, \ldots, a)=1$ and $m_{2}(b, c, a, \ldots, a) \geq-\left(1-\alpha_{2}\right) b$.

Proof of Claim 4. Since $c>b$, by Claim 3 and Lemma 1, it follows that

$$
x_{2}(b, c, a, \ldots, a)=x_{2}(b, b, a, \ldots, a)=1
$$

and

$$
m_{2}(b, c, a, \ldots, a)=m_{2}(b, b, a, \ldots, a) \geq-\left(1-\alpha_{2}\right) b,
$$

which are the desired results.

Claim 5. $m_{1}(b, c, a, \ldots, a) \geq \alpha_{1} c$.

Proof of Claim 5. By the same argument as Claim 3, we obtain that

$$
x_{2}(c, c, a, \ldots, a)=1,
$$

that is,

$$
x_{1}(c, c, a, \ldots, a)=0 .
$$

Then, by the ownership lower bound, we have

$$
m_{1}(c, c, a, \ldots, a) \geq \alpha_{1} c .
$$

Since $b<c$, by Lemma 1 , it holds that

$$
m_{1}(b, c, a, \ldots, a)=m_{1}(c, c, a, \ldots, a) \geq \alpha_{1} c,
$$

which is the desired result. 
Claim 6. For any agent $i \neq 1,2, m_{i}(b, c, a, \ldots, a) \geq \alpha_{i} b$.

Proof of Claim 6. Define $V^{*} \subset \Pi_{i \in N} V_{i}$ as follows:

$$
V^{*} \equiv\left\{v \in \Pi_{i \in N} V_{i}: v_{1}=b, v_{2}=c, \text { and } v_{3}, \ldots, v_{n} \in\{a, b\}\right\} .
$$

For any $\ell=0, \ldots, n-2$, define $V(\ell) \subset V^{*}$ as follows:

$$
V(\ell) \equiv\left\{v \in V^{*}: \#\left\{i \in N: v_{i}=a\right\}=\ell\right\} .
$$

We show this claim by the following induction.

1. For any $v \in V(0)$, it holds that $x_{2}(v)=1$, and that for any $i \neq 1,2$, $m_{i}(v) \geq \alpha_{i} b$.

2. If for any $\ell^{\prime}<\ell$ and any $v \in V\left(\ell^{\prime}\right)$, it holds that $x_{2}(v)=1$, and that for any $i \neq 1,2, m_{i}(v) \geq \alpha_{i} b$, then for any $v \in V(\ell)$, it holds that $x_{2}(v)=1$, and that for any $i \neq 1,2, m_{i}(v) \geq \alpha_{i} b$.

The first part.

Note that $V(0)=\{(b, c, b, \ldots, b)\}$. Then, by Lemma 2 , for any $i \neq 2$, it holds that

$$
x_{i}(b, c, b, \ldots, b)=0,
$$

which means that

$$
x_{2}(b, c, b, \ldots, b)=1 .
$$

Then, by the ownership lower bound, for any $i \neq 2$, we also have

$$
m_{i}(b, c, b, \ldots, b) \geq \alpha_{i} b .
$$

These are the desired results.

The second part.

Let $v \in V(\ell)$. Without loss of generality, we may assume that

$$
v=(b, c, \underbrace{a, \ldots, a}_{\ell \text { agents }}, \underbrace{b, \ldots, b}_{n-2-\ell \text { agents }}) .
$$

By Lemma 2 , for any $i=3, \ldots, \ell+2$, it follows that

$$
x_{i}(v)=0,
$$

which means, by the induction hypothesis and strategy-proofness, that

$$
m_{i}(v)=m_{i}\left(v_{i}^{\prime}, v_{-i}\right) \geq \alpha_{i} b,
$$


where $v_{i}^{\prime}=b$. Furthermore, by the ownership lower bound, it follows that

$$
m_{1}(v) \geq \alpha_{1} b-b x_{1}(v)
$$

and

$$
m_{2}(v) \geq \alpha_{2} c-c x_{2}(v),
$$

and that for any $j=\ell+3, \ldots, n$,

$$
m_{j}(v) \geq \alpha_{j} b-b x_{j}(v) .
$$

We show that $x_{2}(v)=1$. Suppose, to the contrary that, $x_{2}(v)=0$. Since, for any $i=3, \ldots, \ell+2, x_{i}(v)=0$, it means that for some $h \in\{1, \ell+3, \ldots, n\}$, $x_{h}(v)=1$. Then, by summing the above inequalities, we have

$$
\begin{aligned}
\sum_{k \in N} m_{k}(v) & \geq \sum_{k \neq 2} \alpha_{k} b+\alpha_{2} c-b x_{1}(v)-c x_{2}(v)-b \sum_{j=\ell+3}^{n} x_{j}(v) \\
& =\sum_{k \neq 2} \alpha_{k} b+\alpha_{2} c-b \\
& =-\alpha_{2} b+\alpha_{2} c \\
& >0
\end{aligned}
$$

which contradicts the feasibility. Thus, it holds that $x_{2}(v)=1$. Then, by inequalities (1) and (2), we also have that for any $i \neq 1,2, m_{i}(v) \geq \alpha_{i} b$.

Therefore, this claim is valid.

Claim 7. $\alpha_{1}=0$, and for any $i \neq 1,2, x_{i}(b, c, a, \ldots, a)=0$ and $m_{i}(b, c, a, \ldots, a)=$ $\alpha_{i} b$.

Proof. By Claim 4, for any $i \neq 1,2$, we have

$$
x_{i}(b, c, a, \ldots, a)=0 .
$$

By Claim 4, we also have

$$
m_{2}(b, c, a, \ldots, a) \geq-\left(1-\alpha_{2}\right) b .
$$

By Claim 5, we have

$$
m_{1}(b, c, a, \ldots, a) \geq \alpha_{1} c .
$$

By Claim 6 , for any $i \neq 1,2$, we have

$$
m_{i}(b, c, a, \ldots, a) \geq \alpha_{i} b .
$$


By summing these inequalities, we obtain that

$$
\sum_{i \in N} m_{i}(b, c, a, \ldots, a) \geq-\alpha_{1} b+\alpha_{1} c=\alpha_{1}(c-b) .
$$

If $\alpha_{1}>0$, then the right-hand side of the above inequality is positive, which contradicts the feasibility. Hence, we must have $\alpha_{1}=0$. If inequality (3) is valid with a strict sign of inequality for some $i \neq 1,2$, then this also means a contradiction to the feasibility. Thus, we have the desired results.

Since, by Claim 7, $\alpha_{1}=0$, in the following, without loss of generality, we also assume that $\alpha_{3}>0$.

Claim 8. For any $i \neq 1,3, x_{i}(b, a, c, a, \ldots, a)=0$ and $m_{i}(b, a, c, a, \ldots, a)=$ $\alpha_{i} b$.

Proof. By replacing the roles of agent 2 and agent 3 in Claims 3 to 7, symmetric arguments imply the desired results.

Claim 9. We derive a contradiction.

Proof. Since the object is indivisible, it obviously follows that

$$
x_{2}(b, c, c, a \ldots, a)=0 \text { or } x_{3}(b, c, c, a \ldots, a)=0 .
$$

Without loss of generality, we assume that

$$
x_{3}(b, c, c, a \ldots, a)=0 .
$$

Then, by Claim 7 and strategy-proofness, it holds that

$$
m_{3}(b, c, c, a \ldots, a)=m_{3}(b, c, a, a \ldots, a)=\alpha_{3} b .
$$

However, since the ownership lower bound requires that

$$
m_{3}(b, c, c, a \ldots, a) \geq \alpha_{3} c>\alpha_{3} b,
$$

we have a contradiction. ${ }^{11}$

Therefore, Theorem 1 is valid.

Acknowledgments The authors are very grateful to William Thomson, whose lecture at Osaka University on bankruptcy problems motivated us to analyze this topic. The authors also thank Yu Chen, Tomoya Kazumura, Shuhei Morimoto, and Kohei Shiozawa for their helpful comments. This work was supported by JSPS KAKENHI Grant Number 26780117.

\footnotetext{
${ }^{11}$ If we assume that $x_{3}(b, c, c, a \ldots, a)=0$, then we derive a contradiction by Claim 8 instead of Claim 7.
} 


\section{References}

Ando, K., M. Kato, S. Ohseto, 2008, Strategy-Proof and Symmetric Allocation of an Indivisible Good. Mathematical Social Sciences 55, 14-23.

Barberà, S., 2001, An Introduction to Strategy-Proof Social Choice Functions. Social Choice and Welfare 18, 619-653.

Barberà, S., 2012, Strategyproof Social Choice. In: K.J. Arrow, A. Sen, K. Suzumura (eds.) Handbook of Social Choice and Welfare Vol. 2, Elsevier, Amsterdam, 729-831.

Cramton, P., R. Gibbons, P. Klemperer, 1987, Dissolving a Partnership Efficiently. Econometrica 55, 615-632.

Dubins, L.E., E.H. Spanier, 1961, How to Cut a Cake Fairly. American Mathematical Monthly 68, 1-17.

Fujinaka, Y., 2008, A Bayesian Incentive Compatible Mechanism for Fair Division. mimeo.

Gibbard, A., 1973, Manipulation of Voting Schemes: A General Result. Econometrica 41, 587-601.

Hashimoto, K., 2015, Strategy-Proof Rule in Probabilistic Allocation Problem of an Indivisible Good and Money. mimeo.

Holmström, B., 1979, Groves' Scheme on Restricted Domains. Econometrica 47, 1137-1144.

Kato, M., S. Ohseto, S. Tamura, 2015, Strategy-Proofness Versus Symmetry in Economies with an Indivisible Good and Money. International Journal of Game Theory 44, 195-207.

Moulin, H., 1985, Egalitarianism and Utilitarianism in Quasi-Linear Bargaining. Econometrica 53, 49-67.

Moulin, H., 1992, An Application of the Shapley Value to Fair Division with Money. Econometrica 60, 1331-1349.

Moulin, H., 2002, Axiomatic Cost and Surplus-Sharing. In: K.J. Arrow, A. Sen, K. Suzumura (eds.) Handbook of Social Choice and Welfare Vol. 1, Elsevier, Amsterdam, 289-357.

Moulin, H., 2010, Auctioning or Assigning an Object: Some Remarkable VCG Mechanisms. Social Choice and Welfare 34, 193-216. 
Ohseto, S., 2000, Strategy-Proof and Efficient Allocation of an Indivisible Good on Finitely Restricted Preference Domains. International Journal of Game Theory 29, 365-374.

Porter, R., Y. Shoham, M. Tennenholtz, 2004, Fair Imposition. Journal of Economic Theory 118, 209-228.

Sakai, T., 2012, Fair Waste Pricing: An Axiomatic Analysis to the NIMBY Problem. Economic Theory 50, 499-521.

Satterthwaite, M.A., 1975, Strategy-Proofness and Arrow's Conditions: Existence and Correspondence Theorems for Voting Procedures and Social Welfare Functions. Journal of Economic Theory 10, 187-217.

Serizawa, S., 2002, Inefficiency of Strategy-Proof Rules for Pure Exchange Economies. Journal of Economic Theory 106, 219-241.

Sprumont, J., 1995, Strategyproof Collective Choice in Economic and Political Environments. Canadian Journal of Economics 28, 68-107.

Steinhaus, H., 1948, The Problem of Fair Division. Econometrica 16, 101-104.

Thomson, W., 2003, Axiomatic and Game-Theoretic Analysis of Bankruptcy and Taxation Problems: A Survey. Mathematical Social Sciences 45, 249-297.

Thomson, W., 2014, Axiomatic and Game-Theoretic Analysis of Bankruptcy and Taxation Problems: An Update. mimeo.

Zhou, L., 1990, On a Conjecture by Gale about One-Sided Matching Problems. Journal of Economic Theory 52, 123-135. 